\title{
$e=$
}

Earth Common Journal

Changing Climates: Social, Political, Economic

MacEwan University

Volume 6, Number 1, October 2016

Feature

\section{Nutritional Communications across Climates: A comparative research study between Ecuador and the Netherlands}

\author{
Morgan Messelink, MacEwan University, Canada*
}

\begin{abstract}
A study in May 2014 analyzed food labels in Quito, Ecuador, to better understand the culture's nutritional communication. The study explored what is considered to be a healthy diet in Ecuadorian culture and how this is communicated, and also to what extent nutrients in packaged food are effectively communicated via labelling. Data was gathered using a mixed methods approach; first using quantitative methods with a survey administrated to students at the Universidad San Francisco de Quito. Following the completion of the survey, participants were then asked to volunteer for a questionnaire containing openended questions, administered in one-on-one interviews, in order to collect qualitative data to enhance survey responses. Finally, an analysis of nutritional labels in local grocery store completed the research. This same study was then conducted in May of 2016 at Hanze University of Applied Sciences in Groningen, Netherlands, to explore the results from another country and act as a comparative study between the two cultures. Research from both cultures led to the identification of similar and different trends, themes, and outliers in the collected data. Both Ecuador and Dutch participants report receiving little to no formal education regarding diet and nutrition. This leads to participants building their model of a healthy diet from various inconsistent sources. Participants also express frustration and confusion with inconsistent labelling. Simple and measurable food labels in the Netherlands proved to have more importance and value to consumers than labels that are believed to hold false claims.
\end{abstract}




\section{Introduction}

After a study in May 2014 in Quito, Ecuador was completed to better understand nutritional communication and its effect on consumer behaviour, the same research study was completed in Groningen, Netherlands in May 2016 to serve as a comparative study between the two cultures.

Data was gathered using a mixed methods approach; first using quantitative methods with a survey administrated to students at Hanze University of Applied Sciences in Groningen, Netherlands. Following the completion of the survey, participants were then asked to volunteer for a questionnaire containing open-ended questions, administered in one-on-one interviews, in order to collect qualitative data to enhance survey responses. Finally, an analysis of nutritional labels in local grocery store completed the research.

Travelling to both countries to be submerged in the separate cultures and conduct hands on research contributes to the credibility of this research by experiencing nutritional information and communication first hand. Some major findings were found and discussed from the Netherlands and were then used in comparison to the Ecuador results from 2014. Conclusions and recommendations on nutrition communication arose for both cultures and are laid out in this report.

\section{Methodology and Sample Size}

Similar to the research completed in Ecuador, the Netherlands study consisted of a mixed methods approach. In this sequential explanatory design, data was gathered using quantitative methods first, succeeded by qualitative methods. The research began with a survey handed out to students at Hanze University of Applied Sciences in Groningen, Netherlands. The survey consisted of 10 questions, all using a Likert scale for responses. The questionnaire questions were designed using only close-ended questions regarding demographics, instruction received, and consumer behaviour. It intended to obtain a general look at the quality of nutritional information available to students and to determine if it is communicated effectively or not. Following the survey, students had the option to participate in a one-on-one administrated questionnaire. The interview part of the research gathered data using qualitative methods in order to enhance survey questions and to get a better understanding of the value of nutrition information and communication within the Dutch culture. Finally, the data-gathering portion of the study concludes with the quantitative method of content analysis in the examination and analysis of nutrition labels. The data acquired from the label analysis helps to forms the foundation of researchers' 
knowledge on how nutrition is communicated to consumers via front of pack and back of pack food labels, facilitating the determination of the effectiveness of this communication.

In Ecuador, 71 surveys were analyzed and 10 interviews took place. All surveys analyzed included participants between the ages of 18 and 25 years old. To stay consistent, the participants from Netherlands were in the same age bracket with the exception of two participants who were supermarket managers. Those supermarket managers were interviewed and their results included in the interview findings but their surveys were excluded from the total survey results. A total of 80 surveys were completed and returned for analysis in the Netherlands. The two surveys from the supermarket managers were omitted to stay consistent with the intended age range for the survey results. When the surveys were being analyzed, it was found that six of the participants were 17 years of age. These six surveys were also omitted to stay consistent with demographics leaving a total sample size of 72 surveys completed from Hanze University of Applied Sciences. The questionnaire part of the research in the Netherlands consisted of seven participants: five students and two supermarket managers.

Ecuador participants of the survey were 51 per cent (36) female and 49 per cent (35) male, while the interview sample was comprised of an equal number of male and female participants (5 each). Dutch participants of the survey were 46 per cent female (36) and 54 per cent male (42) and interview participants were 3 females and 4 males.

\section{Netherlands Results}

After analyzing the research collected at Hanze University of Applied Sciences and the local grocery franchises in the Netherlands, the following results began to answer the questions: How is nutrition information communicated in the Netherlands, and how does this base knowledge contribute to consumer behaviour?

\section{Nutritional Education}

First major finding was that all interview participants claimed they had received no formal teaching on nutrition or diet. This leads to the belief that the Netherlands has nothing in place for formal education on nutrition in Dutch school system. However, my first interview participant proved this was not the truth. When asked if the participant had any formal education regarding diet or nutrition, they declined and replied that he learned his knowledge of nutrition from "mostly what's on the packages of the products." When the Canadian Food Guide was mentioned in the interview, the interviewee told me about the Schijf van Vijf: the Dutch equivalent to the Canadian Food Guide. The Schijf van Vijf outlines the Dutch dietary guidelines in a format similar to the Canadian Food Guide. The 
interview participant did not, however, bring the Schijf van Vijf up on his own terms, only when asked if there was anything similar to the Canadian Food Guide, and was not mentioned till the end of the interview.

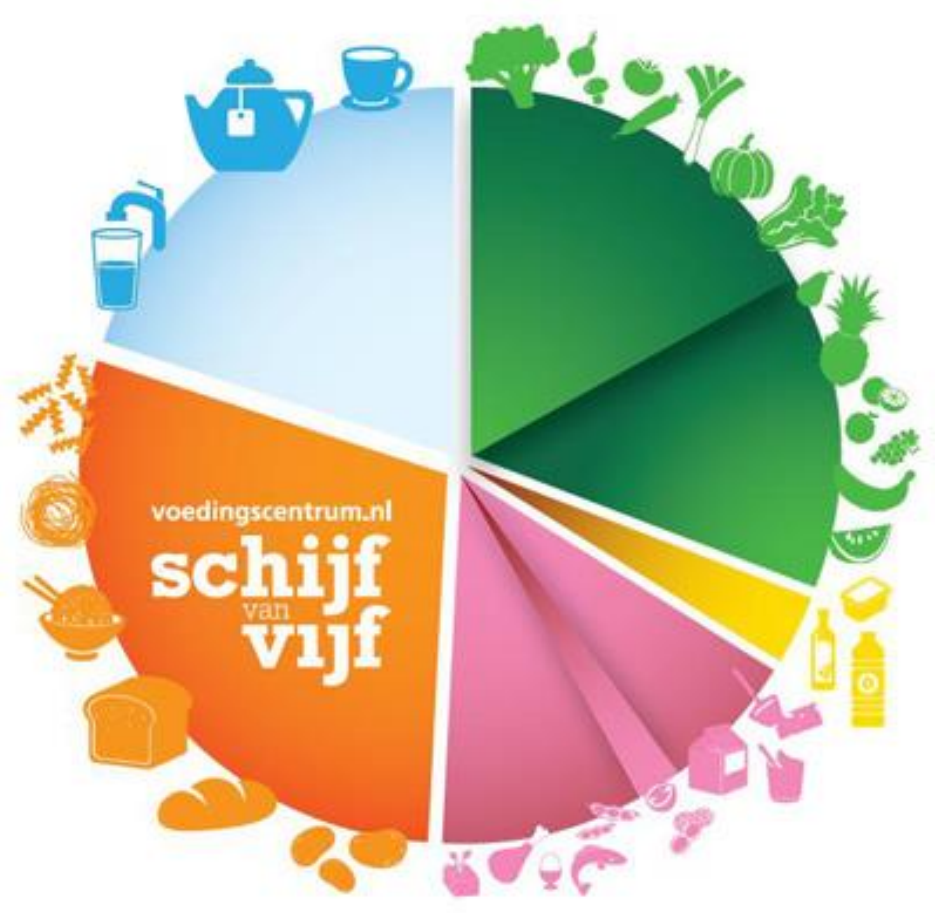

Figure 1. The Schijf van Vijf represents the Dutch equivalent of the Canadian Food Guide.

In all the interviews to follow, the Schijf van Vijf was mentioned to participants. Although, all interviewees initially reported receiving no formal education regarding nutrition or diet, when asked about the Schijf van Vijf all participants were able to identify and recall it. Most claimed they were taught it briefly in elementary school, yet no one mentioned it when initially asked about formal education regarding nutrition or diet. This indicates a significant communication failure between what was taught in school about nutrition and what is actually used as a reference for the basis of a healthy diet. Not one interview participant claimed they followed the Schijf van Vijf or mentioned it without being asked about it. This revealed a missed opportunity by the Dutch government to 
reinforce the Schijf van Vijf as a nutritional guide for citizens to follow when making healthy choices about their diet.

Furthermore, all participants' description of a day-to-day healthy diet was inconsistent with one another. Participants were asked to describe what they thought a healthy diet consisted of and none of the answers followed concrete guidelines or were similar to one another. This supports the notion that no standard guideline on nutrition and diet is being applied, taken seriously, or communicated effectively in Netherlands.

\section{Front of Pack Labelling (FOP)}

Another major finding arising in Netherlands was that front of pack nutritional claims on food products had little to no impact on consumer choice. When asked if a higher nutritional value made it more likely for the participant to select a product, 83 per cent of participants answered yes. But when participants were asked if phrases on food labels like "high in fibre," "low in fat," or "sugar free" played any role in consumer choice, all interview participants expressed they had minimal to no value at all in affecting consumer choice. Three of the seven participants believed that these phrases and symbols on the front of pack labelling could be purchased by manufacturers to help sell their product and therefore disregarded their credit claim altogether. When asked about these claims, participants answered with "I would never buy a light product", "I don't want too much light products where the sugar is replaced," "I ignore front of pack labels", and "I don't take it seriously because I believe companies can buy these things."

These findings are also supported by a study conducted by the Dutch Consumers' Association (2016) where they surveyed over a thousand participants on the effectiveness of the check mark label. They found that 77 per cent of participants did not know exactly what the logos were and 85 per cent did not know the difference between the green (healthier choice) and blue logo (conscious choice) ("Resultaten Panelonderzoek"). The green check mark is for foods products included in the Schijf van Vijf and the blue check mark is for other products. The check mark label indicates if a food product is healthy within its food category. For example, cooking oil may receive a check mark even though frying foods is typically not considered healthy eating. This research found that all participants disregarded the check mark label and believed that it was not of much value. When examining a food product we can see that there may be some truth here. Chocomel Mager, a popular chocolate milk drink in the Netherlands has a check mark logo but also contains six teaspoons of sugar per cup. When following a healthy diet it is commonly known to limit sugary beverages but if consumers are following the check mark logo they may be misguided and misinformed. 

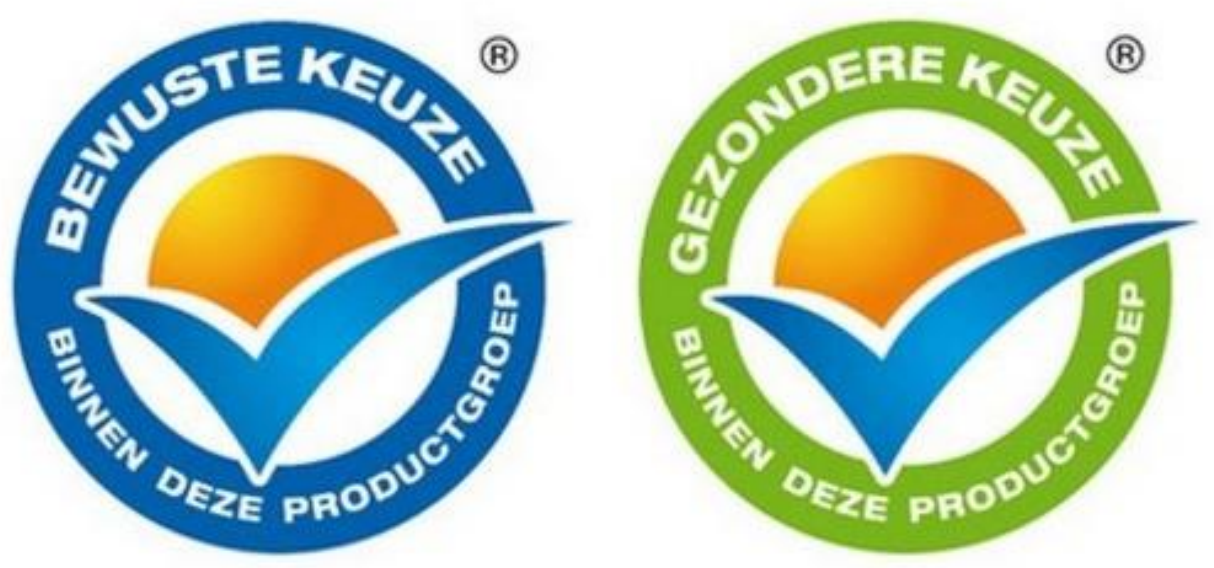

Figure 2. Shows two Dutch front of pack labels. "Bewuste keuze" translates to "conscious choice" and "gezondere keuze" translates to "healthier choice" in English.

\section{“E” Numbers}

Interview participants also expressed confusion and frustration with $\mathrm{E}$ numbers listed in the ingredient list. E numbers are codes for substances that have been approved by the European Union as food additives. E numbers are listed in the ingredient list and require the consumer to look up the $\mathrm{E}$ number if they want to know what it stands for. One participant explained, "I think it's really frustrating because most of the time there are E numbers on there and if I want to know what these things are I need to look it up on Wikipedia and look up everything on that. And so that's really frustrating for me because if I look at the product I just want to know what's in it. So I try to avoid the product with E numbers. It's not very easy to do that." All participants had little understanding of E numbers and one participant had never heard of $\mathrm{E}$ numbers prior to the interview. Although $\mathrm{E}$ numbers make the ingredient list on food labels look shorter and neater, it does not help with communicating nutrition information effectively or simply. Consumers have to manually search each $E$ number to find out what additive it is. This takes time and has proven to be frustrating for research participants and an inconvenience. 


\section{Clearer Labels}

One characteristic of Dutch food labelling that was easy for participants to understand was the Beter Leven symbol on meat products. Beter Leven translates to "better life" in English. The label, found on meat products, communicates to the consumer the quality of life the animal had. The label does that by giving the product one to three stars. The Beter Leven website (beterleven.dierenbescherming.nl) says that animals with the Beter Leven stars "have more space, enrichment material and opportunities to exercise their natural behaviour than animals from conventional livestock." The more stars the product receives, the more ethically it was treated. This label found on meat products in the Netherlands is clear and easily understood. Although, not all interview participants cared about how many stars the animal received, they understood what the labelling was communicating. A clearer labelling system with a measurement aspect proved to be more easily understood to questionnaire participants.

The use of universal symbols is also beneficial for the Beter Leven symbol and its effectiveness. No matter what climate you are in, the idea of three stars versus one star is a universal concept and clearly understood. The Netherlands should consider continuing this shift to using universal symbols in labelling. The purpose of the label can be more easily understood regardless of an individual's language and culture.

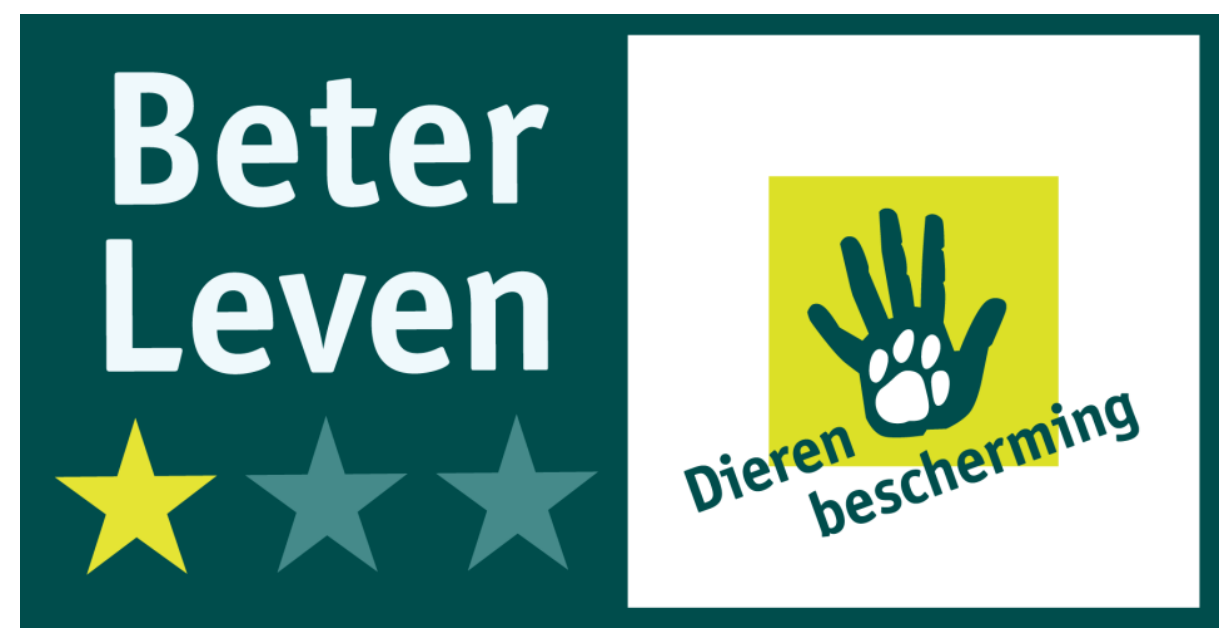

Figure 3. Beter Leven Label. Represents the quality of life an animal had prior to packaging. 


\section{Netherlands and Ecuador: Comparative analysis}

Comparing research results from the Netherlands to those from Ecuador allows similarities and differences of nutrition education and consumer behaviour between the two cultures to be discovered.

\section{Similarities and Dissimilarities}

Survey responses. Similarities between the two cultures were evident when analyzing the survey results. Out of the seven questions that concerned nutritional behaviour (Questions 4 to 10), both countries chose the same majority answer for every question but two. Questions 1 to 3 of the survey were omitted from the table as they were in regards to demographics: gender, age, and highest level of education received. Ecuador participants of the survey were 51 per cent (36) female and 49 per cent (35) male, while the interview sample was comprised of an equal amount of male and female participants (5 each). Dutch participants of the survey were 46 per cent female (36) and 54 per cent male (42) and interview participants were 3 females and 4 males. All participants from both countries were between the ages of 18 and 25 years and all were currently enrolled in postsecondary education. The following table outlines the majority answer from Netherlands results and Ecuador results for the seven interview questions that related to nutrition communication and consumer behaviour:

Table 1.

Summary of Questionnaire Responses

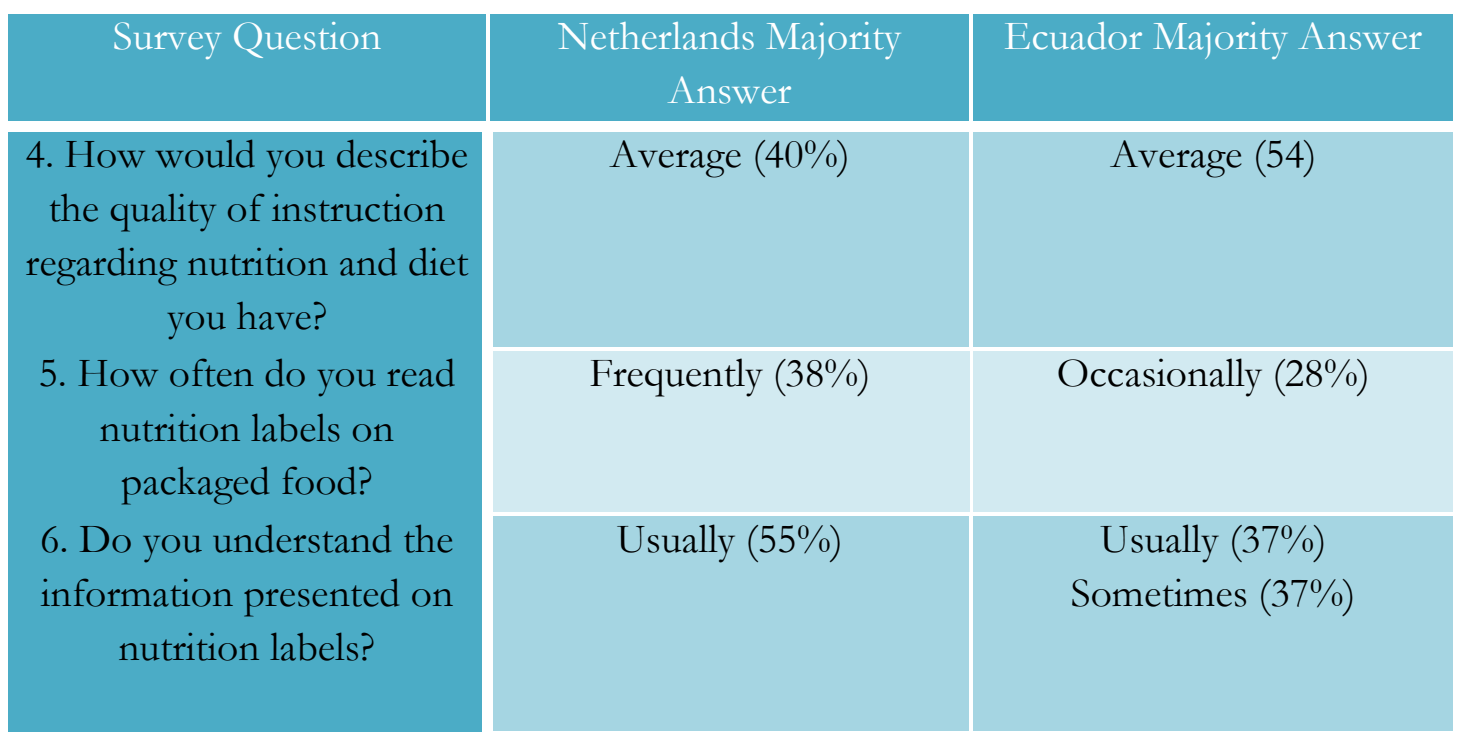




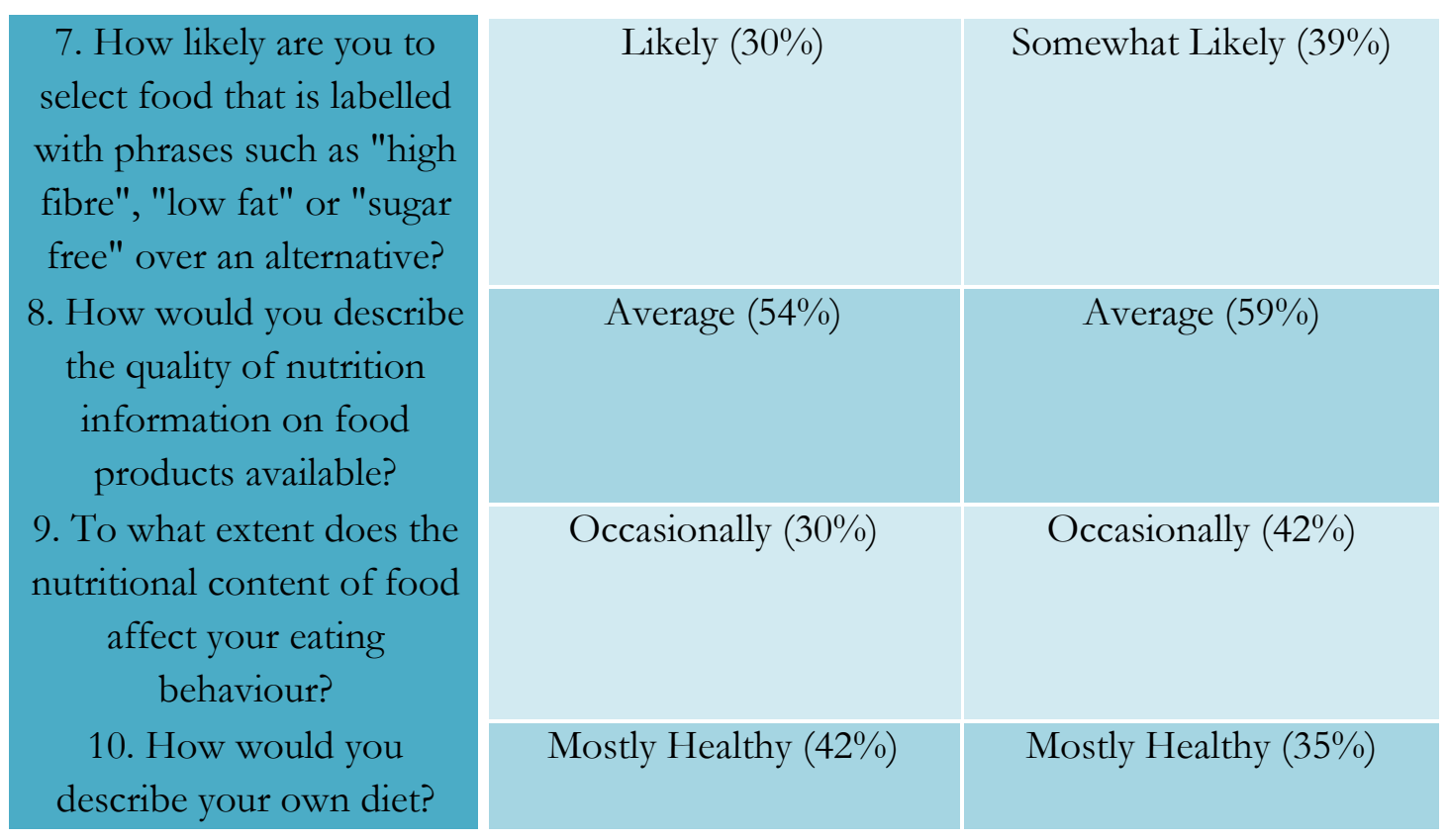

Represents the majority answer to questionnaire questions 4 to 10 from Ecuador participants and Dutch participants. All questionnaire questions were conducted in private one-on-one interviews, and consisted of open-ended questions.

The participants from both countries produced very similar responses to the above questions, concluding that they have a very similar perspective on nutrition and how it is communicated and interpreted in their culture. The majority answers differed in Question 5 (How often do you read nutritional labels on packaged foods?) and Question 7 (How likely are you to select food that is labelled with phrases such as "high fibre", "low fat" or "sugar free" over an alternative?). Netherlands participants answered "frequently" for question five (38 per cent) whereas Ecuador participants answered "occasionally" (28 per cent) as their majority answer. For Question 7, Netherlands participants answered "likely" as their majority answer (30 per cent) whereas Ecuador participants answered "somewhat likely" for their most popular answer (39 per cent). Both Question 5 and 7 are two of the three survey questions that directly address the effectiveness of labels on food products; the other question being Question 6: "do you understand the information presented on nutrition labels?"

Inconsistent labelling. This leads to the similarity that both countries have inconsistent labelling on food products. The research in Ecuador reported that "of the 16 variables analyzed, research showed that only one of the categories (caloric content) was 
available on 100 per cent of the labels gathered, with eight appearing on fewer than 80 per cent and only four appearing on more than 95 per cent of the collected labels" (Adachi, Messelink, \& Pitruniak, 2015, p. 37). Analyzing food labels in the two Dutch grocery stores, six different formats of back of pack food labelling was found. This does not make labelling easy to read or understand and often leads to confusion and frustration for both cultures. One Dutch interview participant commented that "there's so many brands and they are all labeled with different things and pictures, information, and it's just there's so many that I just look at the cheaper one because I don't feel like reading all of them." The labelling has become so overwhelming and confusing for consumers that they disregard labelling all together and just consider price. A grocery store manager commented that customers "don't read their labels, they only look at the price and buy...they have a small income and they don't look if its healthy they just buy, big problem in the Netherlands." If customers are not looking at food labels for their nutritional information then where are they obtaining their basis for a healthy diet?

When in Ecuador, a traffic light system of labelling was sought after. Although Ecuadorian grocery store managers had heard about the implementation of such a labelling system, no products were found with it in place. The traffic light system consists of green, amber and red colour coded front of pack labels. These labels are placed on food products to indicate the level of fat, saturated fat, sugar and salt within; "a 'red' light indicates a 'high' level of that nutrient, an 'amber' light indicates a 'medium' level, and a 'green' light indicates a 'low' level”' (Sacks, Rayner, \& Seinburn, 2009, p. 345). Literature reports that in November of 2013, the Ministry of Health of Ecuador implemented packaged foods to carry 'traffic light' labels ("The traffic," 2014, para. 1). When conducting the initial study in Ecuador, however, no traffic light system labels were found. 


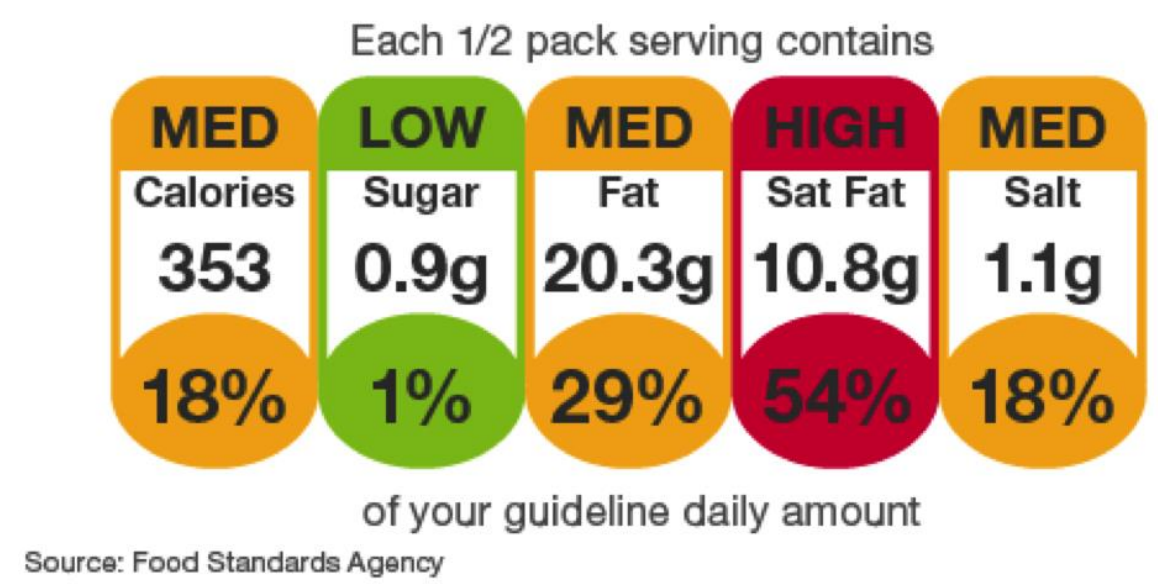

Figure 4. Traffic light labelling for food products.

No traffic light labelling system was found in the Netherlands either, although, there are some are advocating for it. A news article that was released after this research was completed reported that Edith Schippers, a Minister of Health, Welfare, and Sport in the Netherlands, announced that the check mark labels were to be removed and an app was being released that gives consumers personally tailored information about the composition and nutritional value of products. ("App vervangt," 2016). A nutritionist in the article argues that the app is not enough and urges for the introduction of the traffic light labelling in the Netherlands. If Ecuador and the Netherlands both introduced the traffic light labelling they would be successful in implementing universal labelling that transcends climates.

Model for a healthy diet. A major finding from the Ecuador research study in 2014 was the fact that 60 per cent of participants stated that they built their model for a healthy diet from learning at home or by family members. This indicates that in traditional Ecuadorian culture, it is the responsibility of the family to educate their children on the importance of healthy eating, as well as the benefits of following a healthy diet. Meals prepared and served in the home are considered healthy to Ecuadorians, which does not necessarily mean the actual food ingested is meeting all the nutritional requirements. Although there might not be consistency in the nutritional information in Ecuador since one family will differ from another, the source of information is consistent. When Dutch interview participants were asked what they believed to make up a healthy diet, none of the responses were consistent. All interview participants initially recalled no formal education on nutrition or diet and there was no consistency with what actual sources were being used 
to form a model on nutrition or diet. In both cultures, there is no guarantee that participants are actually eating properly based on their sources for constructing a model for healthy eating.

Availability of nutrition information. A final major similarity between Ecuador and Netherlands is that 100 per cent of interview participants from Ecuador research results and 100 per cent of interview participants from Netherlands results agree that the availability of nutritional information could and should be improved. This shows that consumers are not completely satisfied with the current nutrition information availability and stresses the need for improvement.

\section{Conclusions and Recommendations}

Analyzing the results of this research conducted in Ecuador in May 2014 and in the Netherlands in May 2016 revealed correlations and differences in themes, trends, and responses between the two cultures. The results of the surveys, the interviews, and the label analysis all contributed to the discussion of effective nutritional communication and consumer behaviour in the Dutch culture and Ecuadorian culture.

When asked to describe the formal education received regarding diet and nutrition, all Dutch questionnaire participants initially reported receiving none. When asked after to identify the Schijf van Vijf, all participants could successfully do so. This represents a missed opportunity in the Netherlands to reinforce healthy dietary guidelines that will be remembered and respected. In turn, no interview participants reported any concrete guidelines they follow when asked to describe a healthy diet. Ecuador participants reported receiving the majority of their nutrition education from "home." Although it cannot be guaranteed that Ecuadorian home cooked meals are necessarily healthy, it demonstrates that teachings, which have been reinforced over a long period of time, make a lasting impact on Ecuadorian participants. If the Netherlands reinforced the Schijf van Vijf or other similar dietary guidelines over a long period of time it could lead to healthier habits in the Dutch culture and a model for a healthy diet that is respected and referenced.

When asked about the importance and value of front of pack nutritional claims, specifically the conscious and better choice check mark symbol and phrases such as "low in fat" or "sugar free," 100 per cent of Dutch participants said they either ignore them all together or they do not trust the claim. Many participants believed that light products were not healthy for them and believed that these claims can be purchased by manufacturing companies to help sell their products. These claims have lost their credibility with consumers and are not being communicated effectively, if true or not. After analyzing the 
better life symbols for meat products and its effectiveness on consumers, research participants expressed that this labelling was much easier to understand and was taken as truth. Clearer labels, especially with a value system, demonstrated to be more effective and meaningful for participants of this research study.

Both Ecuador and Dutch research participants expressed frustration with inconsistent systems found in their country. Many Dutch participants even admitted to disregarding nutritional information on food labels all together and choosing products based on just price due to being overwhelmed and confused with inconsistent and crowded labelling. And with all interview participants in both countries agreeing that the availability of nutrition communication and information could and should be improved, it is even more important that implementation of clear and consistent food labelling be worked towards.

Finally, this brings up the need for more universal symbols in labelling for both Ecuador and the Netherlands. If the traffic light system for labeling was implemented in both countries, citizens from either country could easily understand and be familiar with food labelling while residing in the other country. This allows for a greater understanding of nutritional information on a global scale, no matter what the social, economical, or political climate is.

The conclusions made from the completion of this research study in Ecuador and in the Netherlands leads to the following recommendations:

- Formal nutritional guidelines should be reinforced in educational systems so to encourage healthy habits at a young age for individuals which will be practiced and referenced throughout adolescence and into adulthood

- Consider clearer front of pack labelling systems, like the Beter Leven symbol, that can be easily understood so to communicate nutritional information effectively

- Implement standards for consistency of information and the layout on back of pack food labels in both the Netherlands and Ecuador, as both cultures expressed confusing and frustration with current labels

- Consider using more universal symbols for labelling on food products so that the label can be easily understandable for more than just one culture and language

This research study served to analyze nutritional base knowledge, consumer behaviour, and nutrition communication in two cultures and to help determine if nutrition information is being communicated effectively or not. These recommendations can contribute to 
achieving a healthier lifestyle in not just Ecuador and the Netherlands but in any culture. This study also serves as a stepping-stone in further research on the topic of nutrition communication and consumer behaviour in changing climates.

* Author: Morgan Messelink is a recent graduate of the Bachelor of Communication Studies program at MacEwan University in Edmonton, Alberta. Her background and interest in nutrition and communications comes together throughout this research. She now lives in Portland, Oregon.

\section{References}

Adachi, K., Messelink, M., Pitruniak, B. (2015). Ecuador: Communicating to bridge the education gap in nutrition. Earth Common Journal, 5 (1) 34-39.

App vervangt gezondheidsvinkjes op levensmiddelen (App replaces health checkmarks on

foods). (2016, October 19). NOS News. Retrieved from

http:// nos.nl/artikel/2138498-app-vervangt-gezondheidsvinkjes-op-

levensmiddelen.html

Beter Leven (Better life). (2016) Retrieved from

https://beterleven.dierenbescherming.nl/beter-leven

Consumentenbond wil af van 'ik kies bewust'-vinkje (Consumers 'wants to get rid of'

Choices checkmark). (2016, March 21). NOS News. Retrieved from

http:/ / nos.nl/artikel/2094176-consumentenbond-wil-af-van-ik-kies-bewustvinkje.html

Google Translate. (2016). Retrieved from https:/ translate.google.com/

Resultaten Panelonderzoek HET VINKJE. (2016, March). Consumentenbond. Retrieved

from

http://content1a.omroep.nl/urishieldv2/127m6d47fb825d2e534b005809ccd0000

ECJ Volume 6, No. 1, 2016: Changing Climates - Social, Political, Economic 
000.ca9ae955a1f76f2a5cdd29a23c030fc5/nos/docs/210316_consumentenbond.p df

Sacks, G., Rayner, M., \& Swinburn, B. (2009). Impact of front-of- pack 'trafficlight' nutrition labeling on consumer food purchases in the UK. Health Promotion International, 24(4), 344-352. Doi:10.1093/heapro/dap032

The traffic light electronic tag. (2014, February 8). Journal TODAY- News Ecuador.

Retrieved from http://www.hoy.com.ec/noticias-ecuador/la- etiqueta-semaforocon-sistema- electronico-600490.html 